\title{
„Gleichbehandeln ist nicht immer gerecht behandeln": Migrantische Studierende an Schweizer Fachhochschulen im Spannungsfeld von Differenzerfahrungen und Handlungsstrategien
}

\author{
Maritza Le Breton ${ }^{*}$ und Martin Böhnel ${ }^{* *}$
}

\section{Zusammenfassung}

Im Fokus dieses Beitrags stehen Ergebnisse aus einem laufenden Forschungsprojekt zur „Internationalisierung an Fachhochschulen der Deutsch- und Westschweiz". Im ersten Fachhochschulgesetz 1995 wurde die internationale Ausrichtung als Verpflichtung für den neuen praxisorientierten und berufsqualifizierenden Hochschultypus der Fachhochschulen festgehalten. Während die mit der Internationalisierung einhergehenden institutionellen Veränderungen auch an den universitären Hochschulen in der Schweiz noch wenig erforscht sind, fehlen Untersuchungen für die Fachhochschulen bisher noch weitgehend. Mit dieser Forschungslücke beschäftigt sich das Forschungsprojekt. Die Studie befasst sich aus ungleichheitstheoretischer Perspektive mit Rahmenbedingungen und Konsequenzen hochschulischer Internationalisierung unter besonderer Berücksichtigung von Migration und Geschlecht, wobei der Fokus auf den Bereich der Hochschullehre resp. die Studienbedingungen gerichtet wird.

Schlagwörter: Gender und Migration in der Hochschulbildung, migrantische Studierende, Ungleichheitsund Differenzverhältnisse, Hochschul- und Fachhochschulforschung

\section{"Treating equally is not always treating fairly": Migrant students at Swiss Universities of Applied Sciences between experiences of difference and agency}

\section{Abstract}

This article is based on a recent Swiss research project on migrant bachelor students at Universities of Applied Sciences and Universities of Education in the German-speaking and French-speaking part of Switzerland, using the educational fields, inter alia, social work and education. Results of the study highlight the complex interplay of gender, migration or national provenience and further dimensions of social differentiation about students' perception in these fields of education. Starting from an intersectional approach on higher education institutions, this contribution addresses formal/informal factors in the university environment and its impact on male/female students' perception of difference, belonging and study success. This contribution wants to point out the practices which students develop in dealing with experiences of social inequality.

Keywords: Gender and migration in higher education, migrant students, relations of inequality and difference, higher education research

\footnotetext{
${ }^{*}$ Maritza Le Breton (Hauptansprechpartnerin): Fachhochschule Nordwestschweiz FHNW, Hochschule für Soziale Arbeit. E-Mail: maritza.lebreton@fhnw.ch

${ }^{*}$ Martin Böhnel, Fachhochschule Nordwestschweiz FHNW, Hochschule für Soziale Arbeit.

E-Mail:martin.boehnel@fhnw.ch

Das vom Schweizerischen Nationalfonds finanzierte Projekt „Internationalisierung an Fachhochschulen: Zur Bedeutung von Geschlecht und Migration für Bildungs(un)gleichheit" wurde in Kooperation mit der Hochschule für Soziale Arbeit (HSA), der Hochschule für Angewandte Psychologie (APS) und der Pädagogischen Hochschule (PH) der Fachhochschule Nordwestschweiz FHNW durchgeführt. Das Projekt startete im September 2016 und dauerte bis 30.04.2019. Neben dem in diesem Beitrag vorgestellten qualitativen Untersuchungsbereich beinhaltete das Projekt ebenso eine Expert*innenerhebung mit Internationalisierungsbeauftragten und Studiengangsleitenden sowie eine Studierendenbefragung (Vollerhebung) in den vier untersuchten Fachbereichen Soziale Arbeit, Pädagogik, Technik und IT sowie Wirtschaft und Dienstleistungen.
} 


\section{Einleitung}

Fachhochschulen in der Schweiz sind aktuell in mindestens zweierlei Hinsicht von Internationalisierungsdynamiken geprägt. Sie bauen einerseits ihre Beteiligung an länderübergreifenden Bildungs- und Austauschprogrammen in Forschung und Lehre zuweilen zögerlich, aber doch stetig aus. Andererseits bieten Fachhochschulen in wachsendem Maße Raum für internationale akademische Belegschaften und für Studierende mit entsprechendem Bildungs- und Herkunftshintergrund.

Die Internationalisierung der Hochschulen bzw. deren Analyse wird zunehmend von Gerechtigkeitsdiskursen begleitet (Lindt/Löther 2008; Fellenberg 2011). Bereits seit den 1970er-Jahren werden Ungleichheiten hinsichtlich Zugangschancen zum Hochschulstudium soziologisch erfasst (z.B. Bourdieu/Passeron 1971). $\mathrm{Ab}$ den 1980er-Jahren und unter Berücksichtigung der Entwicklung der Genderstudies rückten zudem geschlechtsspezifische Differenzen in den analytischen Fokus (z.B. Majcher/Zimmer 2010; Metz-Göckel 2012). Im Zusammenhang mit dem Bologna-Prozess wurde die Frage aktuell, ob und inwiefern diese tiefgreifende Hochschulreform von (Un-)Gleichstellungspotenzialen im Geschlechterverhältnis begleitet sei. Auf konzeptioneller Ebene wurden verschiedene Potenziale identifiziert. Dazu gehören angemessenere Studierbarkeit (Vereinbarkeit des Studiums mit beruflichen, sozialen und familiären Verpflichtungen und individuelle Arbeitsbelastung bzw. die damit verbundenen Beweggründe für Studienabbrüche) durch vorteilhaftere Möglichkeiten des Teilzeitstudiums und der Aufwertung der Lehre (Hering/Kruse 2004). Banscherus et al. (2011: 152) kommen in dieser Hinsicht zu einem negativen Fazit und unterstreichen, die soziale Dimension sei weiterhin als „blinder Fleck des Bologna-Prozesses“ zu betrachten, es sei zudem keine konsistente Strategie in Richtung Überwindung der Ungleichheiten zu erkennen.

Einer vergleichenden Untersuchung zur sozialen Lage der Studierenden in 23 europäischen Ländern ist zu entnehmen, dass die Bildungschancen im Europäischen Hochschulraum ungleich verteilt sind. Abgesehen von den Niederlanden und Spanien, wo Personen aus sogenannten bildungsfernen Familien leicht überproportional in Hochschulen eintreten, sind in beinahe allen untersuchten Ländern Studierende, deren Väter über ein hohes Bildungsniveau verfügen, an Hochschulen deutlich überrepräsentiert (ebd.: 143). Die soziale Selektivität beim Hochschulzugang lässt sich auf Stu- dierende mit Migrationshintergrund übertragen (z.B. Murdoch et al. 2014; Hadjar/Hupka-Brunner 2013).

Auch für den schweizerischen Kontext zeigen verschiedene Studien, dass eine umfassende Chancengleichheit weiterhin „ein nicht realisiertes Desiderat“ darstellt (Stamm et al. 2009: 2 sowie Saner et al. 2016; Felouzis/Charmillot 2017). Die Längsschnittuntersuchung TREE thematisiert in Bezug auf die Primar- und Sekundarstufe den Einfluss der sozialen Herkunft und Migration auf die berufliche Karriere und stellt dabei eine klare Benachteiligung von Jugendlichen mit Migrationshintergrund fest (Scharenberg et al. 2016; Blumer 2016). So kommen die Autor*innen zum Schluss: „Während ein Migrationshintergrund den Zugang zu tertiärer Bildung in Kanada gar begünstigt, führt er in der Schweiz zu einer klaren Benachteiligung" (Blumer 2016: 26).

Hinsichtlich der Verwobenheit der Kategorien Migration und Geschlecht im Kontext der Transformationsdynamik der Hochschulbildung lässt sich ein großer Forschungsbedarf ausmachen, der insbesondere den - vergleichsweise neuen - Hochschultypus der schweizerischen Fachhochschulen betrifft.

Im Fokus des Beitrags steht die Frage, inwiefern für Studentinnen und Studenten mit Migrationshintergrund im Rahmen eines Fachhochschulstudiums neue Gleichstellungspotenziale oder aber neue Ungleichheiten hervorgehen. Die Einschätzungen der befragten Studierenden stehen im Kontext von Ungleichheitsverhältnissen, die sich insbesondere als Differenzzuordnungen bzw. „Andersheit“ (Othering) manifestieren.

Die empirische Datenbasis bilden 16 leitfadengestützte Interviews - mit jeweils acht migrantischen Studierenden ${ }^{1}$ aus der Sozialen Arbeit und Pädagogik - in Anlehnung an das Verfahren des Problemzentrierten Interviews (PZI) nach Witzel (2000), welche im Kontext des Forschungsprojekts „Internationalisierung an Fachhochschulen“ erhoben und mittels Kodierverfahren der Grounded Theory (Strauss/Corbin 1996) ausgewertet wurden. Anhand dieser qualitativ-empiri-

1 Als Synonym für „Studierende mit Migrationshintergrund“ wird die Bezeichnung „migrantische Studierende“ verwendet. Das Adjektiv "migrantisch“ bezieht sich auf eine Selbstpositionierung im Kontext von Emanzipationsbestrebungen. Dieses Attribut scheint - im Gegensatz zur angeblichen homogenen Herkunft der Mehrheitsgesellschaft - offen für sämtliche Phänomene, die in relativ unbestimmter Form mit Migration in Bezug stehen und somit aufgrund tradierter Wissensbestände und binärer Ordnungsschemata gesellschaftlich als , anders' gelten (Polat/Wienand 2014). 
schen Anlage werden die subjektiven Deutungsmuster und Relevanzstrukturen der befragten Studierenden erfasst bzw. rekonstruiert.

Der Beitrag gliedert sich in zwei Hauptteile: Im ersten Teil werden empirische Grundlagen zur Beteiligung von Studenten und Studentinnen in der Hochschulbildung in der Schweiz präsentiert. Als theoretischer Bezugsrahmen zur Erfassung von Differenzverhältnissen im Fachhochschulkontext wird anschließend der Intersektionalitätsansatz umrissen. Nach der Darstellung des qualitativ-empirischen Vorgehens werden im zweiten Teil die empirischen Ergebnisse der Studie und somit die Erfahrungen migrantischer Studierender thematisiert, welche Differenzund Otheringprozesse sowie Handlungsstrategien nachzeichnen.

\section{Gender und Migration in der Hochschulbil- dung - ein Überblick}

Trotz der in den vergangenen Jahren gestiegenen Beteiligung von Frauen auf allen Studienstufen und einer Annäherung des Studienverhaltens von Frauen und Männern im hochschulischen Kontext in der Schweiz lässt sich nach wie vor eine geschlechtsspezifische Segregation als Nachweis sozialer Ungleichheit, insbesondere im Hinblick auf die Studienwahl feststellen (Maihofer et al. 2013; Liebig et al. 2014).

Frauen und Männer sind nicht nur in den unterschiedlichen Berufsbereichen äußerst ungleich vertreten, sondern nehmen in allen Berufen und Professionen - auch bei gleicher Qualifikation - verschiedene Positionen ein (Teubner 2010). Geschlechtsspezifische Unterschiede sind ebenso an den Fachhochschulen zu erkennen. Aktuelle statistische Analysen zur Schweiz halten fest, dass unabhängig vom Hochschultyp die Studienbeteiligung von Frauen und Männern stark zwischen einzelnen Fachbereichen differiert und sich eine ausgeprägte horizontale Segregation der Geschlechter in vielen Fächern beobachten lässt: An den Fachhochschulen überwiegen Männer in den Fachbereichen „Technik und IT“, „Chemie und Life Sciences“ sowie „Architektur, Bau- und Planungswesen“; in den Fachgebieten „Gesundheit“, „Angewandte Linguistik“, „Soziale Arbeit" und „Angewandte Psychologie“ sind Frauen hingegen überrepräsentiert. Auch die Pädagogischen Hochschulen weisen mit 73 Prozent eine große Mehrheit weiblicher Studierender auf (BFS 2017).

Frauen sind aber nicht nur in gewissen Fächern, sondern insbesondere in höheren Stufen der universitären
Ausbildung europaweit unterrepräsentiert (European Commission 2012). Ähnlich wie in anderen Tätigkeitsbereichen sehen sich Frauen im Hochschulkontext Barrieren gegenüber, die sie daran hindern, in höhere berufliche Positionen aufzusteigen („glass ceiling“ resp. „sticky floor“-Phänomen). Dies hat zur Folge, dass für Studentinnen in den entsprechenden Fachbereichen kaum weibliche Vorbilder zur Verfügung stehen, was wiederum Auswirkungen auf die vertikale Segregation haben kann (z. B. Bieri Buschor et al. 2014). Die Unterrepräsentanz von Frauen in bestimmten Fachbereichen bzw. im Wissenschaftsbetrieb wird in der Geschlechterforschung mit verschiedenen Ansätzen erklärt. So befasst sich der Gleichheits- und Differenzansatz mit Bezug auf die Wohlfahrtsstaat-, Arbeitsmarkt- und Professionsforschung mit der Unterordnung und Marginalisierung von Frauen in der Arbeitswelt (z. B. Wetterer 1995 und 1999). Ausgehend von der Sozialisationsforschung thematisieren makro- und mikro-soziologisch orientierte Ansätze Fragen der Nicht-Entscheidung für einen bestimmten Berufs- oder Karriereweg (Gisbert 2001). Konzepte der Organisationssoziologie gehen auf organisationsstrukturelle Hindernisse ein, die Einstiegs- und Aufstiegsprozesse von Frauen sowie deren Verbleib im Kontext einer Organisation oder Profession erschweren (z. B. Wimbauer 1999).

Eine Reihe von Studien verweist zudem explizit auf die Problematik der Vereinbarkeit von Hochschullaufbahn resp. von Beruf, Bildung und Familie (z. B. Buchmayr/Neissl 2006). Ungeachtet der theoretischen Perspektive lässt sich allerdings mit hoher Evidenz nachweisen, dass Frauen im Wissenschaftsbetrieb resp. an Hochschulen von Ausschlussmechanismen sowie offenen oder subtilen Diskriminierungsprozessen betroffen sind, was sich segregierend und karrierehindernd auswirkt (Majcher/Zimmer 2010: 706f.). Hierbei können Ungleichheitsdimensionen - wie Herkunftsund Migrationshintergrund - die Ausgangsbedingungen sowie die Teilhabe- und Entfaltungsmöglichkeiten im hochschulischen Kontext strukturieren und beeinflussen.

Die gesellschaftliche Wirklichkeit in der Schweiz ist durch Migrationsphänomene geprägt. Dabei stehen nicht nur Zuwanderungsbewegungen, sondern die damit einhergehenden Prozesse angesichts von Sprachund Kulturkonfigurationen sowie Zugehörigkeiten und diskursive Konstruktionen der/des Fremden im Vordergrund, welche häufig durch eine stark defizitäre Perspektive auf Migration und Migrierende geprägt sind (Discher/Plösser 2010). Auch Discher und Plösser 
(2010) verweisen auf die grundlegende Bedeutung des Themenfelds „Migration“ für Bildungsinstitutionen. So lässt sich festhalten, dass Studierende mit Migrationshintergrund ${ }^{2}$ nicht nur in den Nachbarländern, sondern auch an Schweizer Hochschulen unterrepräsentiert sind: im Jahr 2016 betrug ihr Anteil 30 Prozent und liegt folglich unter den 41 Prozent der 18- bis 34-jährigen Personen in der ständigen Wohnbevölkerung der Schweiz (BFS 2017: 23). Fast die Hälfte dieser Studierendengruppe waren Eingewanderte der ersten Generation, Personen also, die im Ausland geboren wurden. Die Mehrheit von ihnen (14 Prozent) kamen nach dem Erwerb der Studienberechtigung in die Schweiz. Die übrigen 10 Prozent sind in der Schweiz geboren und zählen damit zur zweiten Generation. Studierende mit Migrationshintergrund sind mit 33 Prozent an den universitären Hochschulen am stärksten vertreten, an den Fachhochschulen beträgt ihr Anteil 29 Prozent und an den Pädagogischen Hochschulen lediglich 18 Prozent (ebd.). Hinter dem Gesamtanteil an ausländischen FH-Studierenden verbergen sich allerdings erhebliche Unterschiede hinsichtlich einzelner Fachbereiche: So liegt ihr Anteil im Fachbereich Musik, Theater und andere Künste bei 45 Prozent, im Bereich Soziale Arbeit hingegen bei 20 Prozent (ebd.: 24f.).

Die ungleiche Beteiligung an tertiärer Bildung ist jedoch nicht lediglich auf einen Migrationshintergrund zurückzuführen, denn das Bildungssystem ist auch durch schicht- und geschlechtsspezifische Differenzen charakterisiert (Becker 2011; Burger et al. 2013;

2 Das Bundesamt für Statistik unterscheidet „Studierende mit Migrationshintergrund" nach Zuwandernden-Generationen und folglich zwischen erster und zweiter Generation (BFS 2015: 25): „Studierende mit Migrationshintergrund erster Generation sind selbst im Ausland geboren. Zur zweiten Generation zählen Studierende, deren Eltern bereits in die Schweiz eingewandert sind, also Studierende, die im Inland geboren sind, aber deren Eltern im Ausland geboren wurden." Die erste Generation wird wiederum in zwei Gruppen unterteilt, nämlich in jene Studierende, die ihre Studienberechtigung in der Schweiz erworben und folglich einen Teil des hiesigen Schulsystems durchlaufen haben, und solche mit einem ausländischen Zulassungsausweis. Diese sind in der Regel zur Studienaufnahme in die Schweiz migriert (ebd.). Auch wenn Geschlecht und Migration gesellschaftliche Konstrukte darstellen, die u.a. durch strukturelle und institutionelle Kontexte bzw. die dazugehörigen Diskurse erst hervorgebracht werden, schließt diese Studie im Sinne einer operationalen Eingrenzung an die hier aufgeführte Definition des BFS an: D.h. die Verwendung des Begriffes Migrationshintergrund bezieht sich hier auf Studierende der ersten und zweiten Generation.
Krüger et al. 2011). Aufgrund spezifischer Zuwanderungsbiografien korrelieren häufig nationale Herkunft resp. Migrationshintergrund und sozioökonomischer Status. „Dies hat zur Folge, dass MigrantInnen und ihre Nachkommen häufiger von schichtspezifischer Benachteiligung betroffen sind. Die Verschränkung von Migrationshintergrund und Schichtzugehörigkeit dokumentiert sich darin, dass Studierende mit Migrationshintergrund häufiger aus einer niedrigen sozialen Herkunftsgruppe kommen als solche ohne Migrationshintergrund" (Burger et al. 2013: 40). Auch die BFS-Studie (2017) macht die Bedeutung der sozialen Herkunft deutlich: der Anteil der Studierenden in der Schweiz, bei denen mindestens ein Elternteil über einen Abschluss auf tertiärer Stufe (höhere Berufsbildung und Hochschule) verfügt, beträgt insgesamt 58 Prozent. Es ist jedoch zu betonen, dass Studierende mit Migrationshintergrund - gemessen am höchsten Bildungsabschluss der Eltern - sich je nach Herkunftsland in ihrer sozialen Herkunft stark unterscheiden (ebd.).

Demnach kann nicht von fehlenden Kompetenzen seitens der Studierenden mit Migrationshintergrund ausgegangen werden. Vielmehr wird in der Literatur die fehlende Passung zwischen den Ressourcen der Studierenden und den Anforderungen des Bildungssystems angeführt, was Probleme und Benachteiligungen nach sich zieht (z. B. Discher/Plösser 2010: 1). Dabei werden insbesondere die Normalitätsanforderungen des Bildungssystems hervorgehoben, die den heterogenen Lebenswelten der Subjekte nur teilweise gerecht werden. Deshalb ist „auch die Hochschule (...) aufgefordert, ihre Funktion als Ort des Ausschlusses und der Benachteiligung von Studierenden mit Migrationshintergrund und der Stabilisierung von Ungleichheitsverhältnissen vor dem Hintergrund nur scheinbar gleicher Ausgangsverhältnisse zu hinterfragen. $\mathrm{Zu}$ reflektieren ist dabei auch, welchen Normalitätskonstruktionen die Hochschule obliegt und wie sie mit Differenzverhältnissen umgeht“ (ebd.: 2).

\section{Intersektionalität als theoretischer Anknüpfungspunkt - Ein Umriss}

Seit den 1980er-Jahren setzt sich in der deutschsprachigen soziologischen Ungleichheitsforschung die Erkenntnis durch, dass soziale Ungleichheit keineswegs das Resultat einzelner sozialer Strukturkategorien wie Klasse oder Schicht darstellt, sondern durch vielfältige Formen und verschiedene Ungleichheitskategorien determiniert wird. So betont bspw. die Frauen- und 
Geschlechterforschung die Bedeutung von Geschlecht als Dimension sozialer Ungleichheit, die Migrationsforschung bezieht sich auf die ungleichheitsgenerierenden Folgen von Rassisierungs- und/oder Ethnisierungsprozessen, und die Altersforschung konzentriert sich auf die Kategorie Alter(n). Seit rund 30 Jahren erfährt diese Debatte theoretische Reflexionen und methodologische Präzisierungen, insbesondere aus der angloamerikanischen Frauen- und Geschlechterforschung, die in ihrer Gesellschaftsanalyse diese Ungleichheitskategorien in ihren Wechselwirkungen auch theoretisch zu erfassen suchte und diese Forschungsperspektive als „Intersektionalität“ bezeichnete. Zwar stellt die an Intersektionalität orientierte empirische Forschung für die Sozialwissenschaften keinen neuen Forschungsansatz dar, da diese Debatte im deutschsprachigen Raum von Schwarzen Deutschen und Diaspora-Feministinnen insbesondere in den 1980er- und 1990er-Jahren angetrieben wurde (Gutiérrez Rodríguez 2011: 77). Zu berücksichtigen sind bspw. die Verschränkung von Kategorien wie Klasse, Geschlecht und Alter in qualitativ und quantitativ angelegten Analysen. Neu sind aber die theoretischen und methodologischen Anstrengungen um Beschreibung und Erläuterung der Interaktionen und Interdependenzen der einzelnen Kategorien jenseits eines bloßen additiven Nebeneinanders. Die mehrdimensionale Analyse sozialer Ungleichheit stellt unter dem Begriff der Intersektionalität in den letzten Jahren die wohl größte Innovation in diesem Feld dar (Winker/Degele 2009).

Grundsätzlich eröffnen Überlegungen zur Intersektionalität weiterführende Perspektiven (Crenshaw 1994; Klinger et al. 2007; Lutz 2007; Lutz et al. 2013; Winker/Degele 2009). Denn sie verweisen nicht nur auf die Bedeutung verschiedener Strukturkategorien, die in ihrer Summe strukturell spezifische Differenzierungen und Ungleichheiten generieren, sondern auch auf die Relationalität und Prozessualität dieser kategorialen Zuweisungen (z. B. Gutiérrez Rodríguez 2011; Lenz 2009; Klinger et al. 2007; Lutz et al. 2013) sowie ihrer wechselseitigen Bedingtheit im Kontext heterogener gesellschaftlicher Verhältnisse und internationaler Machtkonstellationen und daran anschließender Arbeitsteilungen (Dorlin 2010; Falquet 2010). Damit werden Fragen rund um Identitätskonstruktionen resp. Differenzierungspraktiken sowie die strukturellen und institutionellen Kontexte, in denen diese geformt werden, in den Vordergrund gerückt. In Anlehnung an Lutz (2007) lässt sich zudem festhalten, dass Ungleichheitskategorien im Kontext einer inter- sektionalen Perspektive nicht als „essentielle Kategorien“, sondern als Form sozialen Kapitals aufgefasst werden können, welches potenziell in verschiedenen Kontexten unterschiedlich bedeutsam wird. „Ethnizität, Klasse und Geschlecht werden also als Diskriminierung und als Aktionsressourcen relevant" (ebd.: 223). Das Zusammentreffen unterschiedlicher Dimensionen und Differenzlinien kann zu relevanten Interdependenzen führen, die situativ und kontextabhängig Ungleichheit generieren oder gleichstellungswirksam werden. Aus dieser Sicht können also die „Achsen der Ungleichheit“ (Klinger et al. 2007) (wie bspw. Differenzkonstruktionen oder -zuschreibungen hinsichtlich nationaler und geschlechtlicher Zugehörigkeit oder Klassenverhältnisse) im Hochschulwesen nicht notwendigerweise im Sinne von Ausgrenzung, sondern durchaus auch im Sinne biografischer Ressourcen wirken.

Dieser Beitrag nimmt - über den Intersektionalitätsansatz hinaus - Bezug auf weitere Autor*innen, so hinterfragen Bourdieu und Foucault die Angleichung an das „Normale“ oder was als solches (institutionell) legitimiert wird. Mit Bezug auf die Sprache sind bspw. monolinguale Ordnungen Ausdruck von institutionell legitimiertem Wissen bzw. was als „Normativitäten" an untersuchten Hochschulen diskursiv Geltung erfährt.

Konzeptionelle Überlegungen der Studie schließen sich der kritischen Migrationsforschung an und richten sich gegen defizitär orientierte Sichtweisen auf Migration. Dabei werden strukturelle und bildungsbiografische Bedingungen für gelungene Bildungsverläufe in den Vordergrund gestellt, mit dem Ziel, Studienorientierungen und Handlungspraktiken von migrantischen Studierenden mit unterschiedlichen Voraussetzungen im Kontext von Internationalisierungsprozessen an Schweizer Fachhochschulen zu erfassen. Der Intersektionalitätsansatz bietet hierfür eine theoretische Grundlage, um die Vielschichtigkeit von Ungleichheitsdimensionen anzugehen, da sich dieser besonders gut dafür eignet, Mehrfachbenachteiligungen sowie Differenzlinien in ihren gegenseitigen Interdependenzen zu analysieren.

\section{Methodisches Vorgehen}

Als Datengrundlage für die qualitative Untersuchung mit migrantischen Studierenden sind insgesamt 32 Interviews vorgesehen, wobei zu gleichen Teilen Frauen und Männer in den Bachelor-Studiengängen 
Soziale Arbeit, Pädagogik, Technik und IT sowie Wirtschaft und Dienstleistungen an Fachhochschulen der Deutsch- und Westschweiz befragt werden. Die Auswahl der interviewten Studierenden erfolgt nach inhaltlichen Überlegungen resp. soweit möglich entlang kontrastierender Kriterien. Die Zusammenstellung des Samplings orientiert sich somit am Grundsatz, eine möglichst breite Variation der Fälle in der Stichprobe abzubilden, mit dem Ziel, die Vielschichtigkeit und Heterogenität des untersuchten Phänomens zu erfassen. Ausgehend von einer intersektionalen Perspektive werden mit Blick auf den Hochschulkontext und Studierende mit unterschiedlichen Herkunfts- und/oder Bildungsvoraussetzungen folgende Kriterien berücksichtigt: Hochschulstandort, Fachbereich, Studienmodus, Semesteranzahl, Geschlecht, Migrations- und Bildungshintergrund sowie Mehrsprachigkeit. Mit Bezug auf den aktuellen Stand der Datenauswertung finden für vorliegenden Beitrag Aussagen mit 16 migrantischen Studentinnen und Studenten ${ }^{3}$ aus der Sozialen Arbeit und der Pädagogik Berücksichtigung. Die Daten wurden mittels Theoretischem Kodieren in Anlehnung an die Grounded Theory (Strauss/Corbin 1996; Glaser/ Strauss 2005) ausgewertet. Die mit dieser Forschungsperspektive implizierte Notwendigkeit der Offenheit im Forschungsfeld ließ diese Methodologie als besonders passend erscheinen. Die Daten wurden folglich in mehreren Schritten verdichtet: zunächst wurden die Interviews mit Hilfe der Software Atlas.ti offen kodiert sowie für einzelne reichhaltige Interviews eine Fallcharakterisierung erstellt. Diese ermöglichen eine Komprimierung der Daten, indem als analytisches Raster folgende Dimensionen entlang der forschungsleitenden Fragestellungen herangezogen wurden: biografische Verortung, institutionelle Hürden, Handlungsoptionen sowie die Bedeutung von Geschlecht und Migrationserfahrung als mögliche Formen kulturellen und sozialen Kapitals. Die Fallcharakterisierungen wurden im Verlauf der Analyse entlang der thematischen Dimensionen weiter verdichtet. Dies erlaubt, die Interviews in einer vertikalen Analyse zu erschließen, welche in einem weiteren Schritt entlang der thematischen Dimensionen in Verbindung zueinander gesetzt werden. Deren Auswahl stützt sich auf Überlegungen zu intersektionalen Forschungsvorhaben. So soll anhand einer

3 Die Interviews wurden vollständig anonymisiert und mit Pseudonymen versehen. „begründeten Reduktion“ (Pöllmann-Heller/Bitzan 2017: 23f.) mit ausgewählten Differenzkategorien bzw. Migration und Geschlecht zum einen die Bedeutung subjektiver Erfahrungen mit Studienbedingungen erschlossen werden. Weiter ist es Ziel, verschiedene Differenzkategorien in ihren Wechselwirkungen und Bedeutungen für individuelle Handlungsoptionen $\mathrm{zu}$ untersuchen. Überdies können so die Verfestigung bzw. Auflösung von Ungleichheitsstrukturen an den Hochschulen identifiziert werden. Dieses Vorgehen ermöglicht, eine horizontale Analyse empirischer Ausprägungen und Regelmäßigkeiten entlang der Fallcharakterisierungen vorzunehmen und fallübergreifende Zusammenhänge hinsichtlich intersektionaler Verhältnisse zu generieren.

\section{Differenzerfahrungen und Handlungsstrategien aus der Sicht migrantischer Studentinnen und Studenten - ein empirischer Blick}

Die Aussagen der Studierenden decken ein breites Themenfeld ab, geprägt von unterschiedlichen Erfahrungen im Studium, in dessen Verlauf sie sowohl Beteiligte von Differenzerfahrung sind als auch an ihrer Reproduktion mitwirken. Die Ergebnisse der Untersuchung veranschaulichen defizitäre Zuschreibungen angesichts monolingualer Sprachverständnisse und die Unterstellung kultureller Andersheit bspw. seitens Dozierender. So erweist sich die Gleichsetzung von sogenannten muttersprachlichen Kenntnissen mit „perfekten“ Deutsch- bzw. Französischkenntnissen oftmals als symbolische Ordnung der Unterscheidung zwischen „Wir“ und „den“ „Anderen“ und der Aberkennung migrationsspezifischer Sprachvarietäten. Zugleich verdeutlichen Differenzkonstruktionen aufgrund des Aussehens, dass migrantischen Studierenden aufgrund ihres Erscheinungsbilds teilweise mangelnde Sprachkenntnisse unterstellt werden, was sich in ethnisierten Bildern "des“ „Anderen“ niederschlägt. Ältere Studierende äußern zudem Hierarchien in Gruppenbildungsprozessen: So zeigt sich, dass der Altersunterschied oftmals als Ausschlussmoment fungiert, indem sich jüngere Mitstudierende gegenüber älteren Studierenden distanziert verhalten. Im Hinblick auf die Interdependenz unterschiedlicher Differenzen sowie institutioneller Rahmenbedingungen lässt sich festhalten, dass migrantische Studierende wider kategorialer Zuweisungen und ausgelasteter Studien- und Lebenskonstellationen zielorientiert vorgehen. 
5.1 Zur Problematik von Differenz an Fachhochschulen

Die Aussage der Studentin S. Erdal, "gleichbehandeln ist nicht immer gerecht behandeln" (Z. 519-520) problematisiert das an Hochschulen weiterhin vorkommende pädagogische Handeln von Dozierenden, Menschen mit "Migrationshintergrund“ als homogene Gruppe zu erfassen. Die Daten veranschaulichen dies insbesondere durch das Denken in monokulturellen und monolingualen Selbstverständnissen (Kalpaka 2009: 33), was kritisch betrachtet die Gegenwärtigkeit von Migrationsgesellschaften außen vorlässt.

So konstatieren Mecheril/Melter (2010: 117) mit Blick auf die Soziale Arbeit, dass die Herstellung von Differenz und Andersheit auf der Grundlage von Einund Ausschließungspraxen für die Profession bestimmend sind, was auf das Spannungsfeld und implizite Mechanismen des Unterscheidens, Grenzziehens und Normalisierens in der Sozialen Arbeit deutet (Riegel 2016: 96). Solche Mechanismen stellt Riegel (ebd.: 114) auch für den Bildungskontext fest, welche zwischen Gruppen eine hegemoniale Differenzordnung manifestieren können. Insofern verortet sie den differenzgeprägten Blick auf das „Andere“ angesichts einer machtbesetzten sowie „unausgesprochenen ,Normalität', mit dem (pädagogischen) Ziel der Normalisierung und Integration, aber auch verbunden mit der Gefahr der Ausgrenzung und des Othering“" (ebd.: $7 \mathrm{f}$.). Es zeigt sich anhand der Daten, dass migrantischen Studierenden teils aufgrund des Erscheinungsbilds bzw. Aussehens seitens Dozierender mangelnde Sprachkenntnisse unterstellt werden, auch solchen der zweiten Generation. Kalpaka (2009: 33) zufolge werden Differenzen in der "Diskriminierung durch Gleichbehandlung" übersehen, solange nicht gesellschaftlich anerkannt wird, dass migrantische Studierende in der Gesamtheit verfügbarer Ressourcen und Lebensverhältnisse besonderen Schutz bedürfen. Insofern führt die Nichtanerkennung von Differenz vielmehr zur Verfestigung von Ungleichheiten als zur Gleichstellung von Personen (Kalpaka 2002: 40). Damit wird die Frage aufgeworfen, ob der Diskurs zu Heterogenität von migrantischen Studierenden insgesamt als Auseinandersetzung mit Defiziten oder Potenzialen an Schweizer Fachhochschulen geführt wird.

Aussagen der befragten Studentinnen und Studenten verdeutlichen unterschiedliche lebensweltliche Herausforderungen und Benachteiligungen an den untersuchten Fachhochschulen. Auch wenn die
Ursachen von Differenzerfahrung aus der Intersektion unterschiedlicher Dimensionen entstehen, ist die Benachteiligung migrantischer Studierender auf den „Migrationshintergrund“ sowie weitere Aspekte zurückzuführen.

5.2 Sprache als Unterscheidungs- und Distinktionsmerkmal im Studium

Im Rahmen des Zulassungsverfahrens thematisieren migrantische Studierende Differenzerfahrungen aufgrund von sprachlichen Anforderungen. Im Praxisfeld der Sozialen Arbeit gehöre der Nachweis über das Sprachniveau C2 - neben sechsmonatiger Praxiserfahrung - zu den Zulassungsvoraussetzungen des Studiums. Diese setzen im Fall S. Erdals eine hohe finanzielle Investition voraus und werden ihrerseits als große Hürde wahrgenommen, schildert die Studentin: „Das ist ein hohes Sprachniveau und dafür muss man viel Geld investieren, damit man dieses Diplom bekommen kann. Dafür muss man viele Sprachkurse machen, also das finde ich eine hohe Hürde, aber zum Glück konnte ich das schaffen" (Z. 50-54). Dieser Herausforderung begegnet die Studentin durch einen zeitlichen und finanziellen Mehraufwand. Zur Optimierung der Sprachkenntnisse absolviert sie Praktika in mehreren sozialen Einrichtungen. Dies wird von S. Erdal in folgender Interviewsequenz näher beschrieben: „In drei Organisationen habe ich ein Vorpraktikum gemacht. (...) Ich wollte meine Sprachkenntnisse verbessern. Es hätte gereicht, eine Praktikumsstelle, sechs Monate, aber ich habe drei Praktika gemacht" (Z. 26-30).

Analog zur Situation der Studentin S. Erdal, berichtet V. Heger - auch Studentin der Sozialen Arbeit -, dass eine tiefergehende Auseinandersetzung mit der Wissenschaftssprache Deutsch für sie einen Mehraufwand gegenüber Schweizer Studierenden bedeuten würde. Der zeitliche Aspekt ist für sie mit finanziellen Anstrengungen verbunden, da sie von staatlicher Seite keine Unterstützung erhält; mit der Begründung, ihre Deutschkenntnisse würden zur „Lebensbewältigung“ (Z. 84) ausreichen. Die im vorliegenden Fall verantwortliche Sozialbehörde sieht keinen Grund, V. Heger finanziell dabei zu unterstützen, ein höheres Sprachniveau zu erlangen. Ihr Ziel ist es dennoch, wie sie in der folgenden Interviewsequenz ausführt: "Ich wollte ein höheres Niveau, damit ich glücklich bin, aber es wurde entschieden, nein, dies braucht es nicht" (Z. 85-87). Das höhere Niveau verbindet V. Heger mit der Motivation, die Hochschule 
zu besuchen. Der Entscheid, die Studentin nicht zu unterstützen, ist auch eine Absage, in ihr kulturelles Kapital zu investieren. Da die Behörde V. Heger den Hochschulbesuch möglicherweise nicht zutraut, wird sie aufgrund der Sprache als „Andere“ konnotiert und abgesondert.

Die vorgenommene Setzung impliziert, Studierende mit Erstsprache Deutsch vorbehaltlos zur sprachlichen Norm zu definieren. Auf der Basis einer dichotomen Ordnung resp. entgegengesetzten Polen „Wir“ und die „Anderen“ werden seitens der Behörde „befähigte“ Subjekte gebildet und „Andere“ ausgeschlossen (Schwendowius 2015: 101). Dadurch wird markiert, was Foucault einst damit andeutete, wer der „Normalisierungsgesellschaft“ angehören soll: „Eine Normalisierungsgesellschaft ist der historische Effekt einer auf das Leben gerichteten Machttechnologie“, so Foucault (1977: 139) in Der Wille zum Wissen. Darunter ist der nach Foucault zeitliche Wandel zu verstehen, wenn das Funktionieren der Institution immer mehr der Norm unterliegt und „das Lebende in einem Bereich von Wert und Nutzen zu organisieren“ (ebd.) ist. Seit Sexualität und Wahrheit ist sein Verständnis der Institution geprägt, weg von rein disziplinierenden unter Einbezug von neu regulierenden Kontrollen (ebd.: 135) der Institution über „Körperleistungen und Lebensprozesse" (ebd.). Veranschaulicht an der Situation von V. Heger bedeutet dies, dass die Behörde mittels finanzieller Mechanismen über den Werdegang der Studentin zu bestimmen versucht, deren Maßnahmen zudem rassisierte Konstruktionen des „Anderen“ aufgrund von Herkunft und Sprache hervorbringen und somit entscheidet, wer an der „Normalisierungsgesellschaft" teilnehmen kann.

Migrantische Studierende berichten zudem über Differenzerfahrung im Rahmen von Gruppenarbeiten, was „normative“ Sprachvoraussetzungen anbelangt. So sind sie teilweise auf die Bereitschaft Schweizer Studierender angewiesen, mit ihnen Hochdeutsch zu sprechen. Aus den Daten ist ersichtlich, dass diese während Gruppendiskussionen oft in den regionalen Dialekt wechseln, der aber nicht von allen migrantischen Studierenden verstanden wird. Es erfordert daher Mut, erzählen zwei Studentinnen, die Kommiliton`innen darauf anzusprechen, ins Hochdeutsche zu wechseln, insbesondere wenn auch nach mehrmaliger Aufforderung im Dialekt weitergesprochen wird. Eine Studentin schildert, sie habe aus solchen Vorkommnissen den Eindruck gewonnen, dass Schweizer Studierende mit dem Sprachwechsel Mühe bekundeten, da diese teilweise selbst "gebrochen Hochdeutsch" ( $Z$. 181) sprächen. So zeigen Ergebnisse eines Projekts zu Mehrsprachigkeit auf, ${ }^{4}$ dass sprachbezogene Probleme sowohl bei Studierenden mit Deutsch als Erstsprache als auch bei denjenigen mit Deutsch als Zweitsprache auftreten, und deshalb „die Unterscheidung zwischen Dialekt und Standardsprache sowie das wissenschaftliche Schreiben - Herausforderungen für fast alle Studierenden darstellen“ (Knappik/Dirim 2013: 21). Diese Ausführungen relativieren die weit verbreitete Annahme, muttersprachliche Kenntnisse seien mit perfekten Sprachkenntnissen gleichzusetzen. Eine Studentin fügt in folgender Interviewsequenz an, wie sie von Schweizer Mitstudierenden aufgrund der Sprache als „Andere“ konnotiert wird: „Meine Schweizer Mitstudierenden können auch nicht alle die Sprache. (...) Sie müssen sich dann jemanden wie mich [Migrantin] heraussuchen, um Gegenbilder zu schaffen" (Z. 639-642).

Den Daten ist ebenso zu entnehmen, dass Dozierende an der untersuchten Hochschule für Soziale Arbeit in der Deutschschweiz die Lehrveranstaltungen teilweise im Dialekt halten. Eine Studentin berichtet dazu: „Es kommt selten vor, aber es gibt immer Dozierende, die möchten einfach auf Dialekt vortragen" ( $Z$. 130-131). Allerdings entgegnet sie, darauf angesprochen, wie sie mit einer solchen Situation umgehe, „dass sie [Dozierende] sich auch selbst ein bisschen die Möglichkeit nehmen, diesem Thema gerecht zu werden" (Z. 135-136).

Studien zu gender- und migrationsspezifischer Diversität aus der Perspektive von Studierenden der Sozialen Arbeit (z. B. Le Breton et al. 2016: 157) halten fest, dass in Gruppenarbeiten die Sprache mit fachlicher Kompetenz und sozialer Anerkennung gleichgesetzt und Zugehörigkeit aufgrund von Sprachkenntnissen verhandelt wird.

Kritische Forschungsperspektiven, die sich für migrationsgesellschaftliche resp. mehrsprachige Verhältnisse aussprechen (Quehl/Mecheril 2008) und mit dem Begriff „migrationsspezifisches Deutsch“ (Knappik et al. 2013: 44) sowohl die Varietät des Deutschen aus Dialekten, Soziolekten und Sprechweisen unter Einfluss von Migrationssprachen gleichwertig betrachten (ebd.), kritisieren Normalitätsvorstellungen, dass von mehrsprachigen Studentinnen und Studenten zu

4 „Diversität und Mehrsprachigkeit in pädagogischen Berufen" ist ein vom österreichischen Bundesministerium für Unterricht, Kunst und Kultur unterstütztes Projekt, welches den Anteil an Lehrpersonen mit Migrationshintergrund an österreichischen Schulen zu erhöhen versucht. 
erwarten sei, perfekt Deutsch zu sprechen (Knappik/ Dirim 2013: 22).

Knappik et al. hinterfragen vorherrschende Annahmen, „dass eine komplette sprachliche Assimilation unter das, was als die native speaker-Varietät des Deutschen angesehen wird, stattfinden muss" (2013: 44). Gerade vor dem Hintergrund eines naturalisierenden Umgangs mit der Wissenschaftssprache Deutsch (Knappik/Dirim 2013: 23) kann auch die hier untersuchte Hochschule für Soziale Arbeit betrachtet werden, wonach Sprachkenntnisse auf der höchsten Stufe des europäischen Referenzrahmens als Zulassungsanforderung gelten. ${ }^{5}$

Insbesondere im monolingualen Hochschulkontext wird die Vorstellung von Studierenden befördert, dass sich als Verständigungsbasis nur die Sprache der Mehrheitsgesellschaft als „legitime Sprache“ (Bourdieu 1990) erweist. Insofern bewirken Differenzordnungen hinsichtlich monolingualer Imaginationen potenzielle Stigmatisierungen auf Studierende, die Deutsch als Zweitsprache erlernt haben. Folgende Interviewsequenz verweist auf die Stigmatisierung seitens Dozierender einer migrantischen Studentin aufgrund angeblicher Sprachdefizite: „Ich sage, woher wissen sie [Dozierende] das, dass ich es schwieriger habe? Mit wem haben sie mich jetzt verglichen? (...) bei mir erwähnen sie die Defizite. (...) Ich denke mir, es gibt noch zigg' Möglichkeiten, wo man Defizite haben kann. (...) Immer zuerst diese Sprache" (Z. 617-623).

Zudem halten Studien den defizitär ausgerichteten Diskurs bei Studierenden mit migrationsbedingter Mehrsprachigkeit fest (Knappik/Dirim 2013; Schwendowius 2015; Schwendowius/Thoma 2016). Eine befragte Studentin der Pädagogik berichtet über ein Vorkommnis in einer Lehrveranstaltung, in der die Dozentin Studierende nach der Anzahl der von ihnen gesprochenen Sprachen im Raum gruppieren lässt. Dabei nimmt die Dozentin zur Illustration der Klassenzusammensetzung Mehrsprachigkeit als Beispiel, um migrationsspezifische Vielfalt in Schweizer Primarschulklassen zu veranschaulichen. Die Dozentin weiß über die Mehrsprachigkeit der Studentin Bescheid und hebt diese gegenüber ihren Kommiliton*innen positiv hervor. Dadurch stellt die Dozentin nicht nur

5 „Studienanwärterinnen und -anwärter, die Deutsch als Zweitsprache sprechen, haben den Nachweis genügender Kenntnisse der Unterrichtssprache für das Bachelor-Studium auf dem Niveau C2 zu erbringen“ (Studienreglement zum Bachelor-Studium an der untersuchten Hochschule für Soziale Arbeit 2015). die sprachliche Kompetenz der Mitstudierenden in Frage. Ihr Handeln kann von Studierenden zum Anlass genommen werden, Mitstudierende nach der Anzahl der von ihnen gesprochenen Sprachen anlässlich einer dichotomen Ordnung zu stigmatisieren.

Die Gegenüberstellung der Eigengruppe durch Essentialisierung resp. kategoriale Zuschreibungen der „Fremdgruppe“ und einhergehenden Legitimierung als „anders“ bezeichnet Attia (2014: 9) als Othering, um zwischen der dominanten „Wir-Gruppe“ und „Migrationsanderen ${ }^{\text {c6 }} \mathrm{zu}$ unterscheiden, was Ein- und Ausgrenzungsprozesse nach sich ziehen kann.

\subsection{Unterstellte Andersheit aufgrund des Erscheinungsbilds und des Alters}

Im Gegensatz zu Schweizer Studierenden repräsentieren migrantische Studierende an Schweizer Fachhochschulen nach wie vor eine Minderheit. Einige scheinen als Vertretende einer minoritären Gruppe im Studium aufzufallen und werden u. a. aufgrund ihres Erscheinungsbilds als „Migrationsandere“ (ebd.) wahrgenommen. Im Fall von Z. Anesani - Studentin der Sozialen Arbeit - werden ihre andere Hautfarbe und der von ihr getragene Turban von einigen Dozierenden mehrfach als Motiv genommen, sie als Repräsentantin einer anderen Kultur zu markieren (Panagiotopoulou et al. 2016). Die andere Hautfarbe und der Turban als Ausdruck von "Andersheit" wird von einem Dozenten zudem als Legitimation aufgefasst, ihr mangelnde Sprachkompetenzen zu unterstellen. So wird ihr, wie folgende Interviewsequenz zeigt, auf Hochdeutsch die Frage gestellt, ob sie den Ausführungen an der Lehrveranstaltung folgen könne: „Verstehen Sie mich? Und nachher, das ist so spannend, dass ein Akademiker nicht davon ausgeht, dass jemand, die so aussieht wie ich, auch hier sozialisiert werden kann. Als hätte er bereits eine feste Vorstellung im Kopf von Menschen mit anderer Hautfarbe" (Z. 227-230). Den Hinweis auf diskursiv zugeschriebene körperliche oder „kulturelle“ Merkmale, also der Reifi-

6 Paul Mecheril (2010:17) verweist mit dem Begriff „Migrationsandere“ darauf, dass Differenzen entlang der binären Konstruktionslogik „Migrant und Nicht-Migrant“ nicht per se existieren, sondern als relationales Phänomen im Kontext der Migrationsgesellschaft zu deuten sind (vgl. dazu auch Discher/Plösser 2010: 4). „Der Terminus ,Migrationsandere' soll nun zum einen die mit dieser Relation einhergehende Festschreibung und Pauschalisierung deutlich anzeigen und zum anderen zur Reflexion des Konstruktionsprozesses von Andersheit auffordern“ (ebd.). 
zierung von Andersheit, beschreiben Mecheril/Melter (2010) als herabwürdigende Unterscheidung zwischen „Wir“ und den „Anderen“ im Kontext der Sozialen Arbeit. Auch V. Heger berichtet, Mitstudentinnen hätten ihr aufgrund ihres „bunten“ (Z. 316) Kleidungsstils politische Inkorrektheit unterstellt, so als würde sie nicht "realisieren, was um sie herum in der Welt passiert" (Z. 318-319). Dadurch werden Konfigurationen von „Andersheit“ aufgrund des Aussehens veranschaulicht. Demnach nehmen Studierende den bunten Kleidungsstil V. Hegers als different wahr und unpassend für ein Studium in Sozialer Arbeit. Zugleich sind bspw. für den Pädagogikstudenten Y. Aslan Sympathie und Erscheinungsbild zentrale Ein- bzw. Ausschlusskriterien für einen bestimmten bevorzugten Lebensstil: „Ich sage nicht, ich gehe nur mit einem Mann reden, weil er das gleiche Geschlecht hat wie ich, sondern eher wegen der Sympathie. Das ist jetzt ein wenig oberflächlich, aber das Aussehen, also wer zieht sich ähnlich an, wer hat einen ähnlichen Stil, ist halt ein Grund, um mich in einer Veranstaltung eher neben ihn zu setzen" (Z. 597-401). Andererseits vermittelt der Kleidungsstil V. Hegers bei ihren Mitstudierenden den Eindruck eines mangelnden sozialpolitischen Bewusstseins, als manifestiere ein bestimmtes Aussehen in der Sozialen Arbeit soziale Kompetenz und politisches Engagement. In folgender Interviewsequenz schildert sie, dass sie auch außerhalb der Hochschule aufgrund des Erscheinungsbilds mit Vorurteilen konfrontiert sei, an der Hochschule erwarte sie jedoch insbesondere seitens Mitstudentinnen eine differenziertere Haltung: „Einfach als Migrantin habe ich bemerkt, dass ich vielleicht durch meine Optische, ah nach aussen durch mein Erscheinungsbild, dass ich dann auch häufig gemerkt habe, dass die Frauen gerade ziemlich Vorurteile haben können. Das hat mich überrascht. (...) Ich bin konfrontiert mit genau den gleichen Vorurteilen wie draussen. Ah, das heisst zum Beispiel, wenn ich vielleicht mehr Farben habe. /Eh/ dass das vielleicht nicht so rüberkommt, dass ich nicht politisch korrekt sein kann. Oder dass ich nicht genug realisiere, was um mich herum in der Welt passiert" (Z. 303-319).

Die hier geschilderte Rhetorik der Auffälligkeit von migrantischen Studierenden mit Blick auf die äußere Erscheinung täuscht das Bild, Studierende der Sozialen Arbeit seien als Akteur*innen von Differenzierungspraxen ausgenommen. Vielmehr wird dadurch verdeutlicht, dass migrantische Studierende den an sie gerichteten Erwartungen und diskriminierenden Äußerungen seitens ihrer Mitstudierenden standhalten müssen.
Zwei befragte Studentinnen aus der Pädagogik berichten über Differenzerfahrung im Studienalltag aufgrund des wahrgenommenen Altersunterschieds seitens jüngerer Mitstudierender, die sich ihnen gegenüber deswegen distanziert zeigen. Deren Alter nehmen jüngere Bachelor-Studierende teils als Barriere wahr, mit ihnen in Kontakt zu treten. So erleben sich ältere Studierende als Minderheit und grenzen sich auch gegenüber jüngeren Studierenden ab. Der Altersunterschied scheint im Studium als Motiv für hierarchische Prozesse und verwehrte soziale Anerkennung zu wirken. C. Durion - Studentin der Pädagogik - bringt die wahrgenommene Distanz wie folgt zum Ausdruck: „Die haben das letzte Jahr das Abi [Matur] gemacht oder dieses Jahr und sind vielleicht maximal 20, 22 oder so. Und dann sind wir einige wenige in meinem Alter, es gibt noch eine, die ist noch älter. Also von daher ist es auch eine Sache, dass die Mitstudenten nicht so recht wissen, wie sie mit mir umgehen sollen" (Z. 405-409). Die Pädagogikstudentin A. Sanda nimmt ihrerseits altersspezifische Hierarchien in Gruppenbildungsprozessen wahr. So erwähnt sie, dass sich bestimmte Studierende bereits während der Studieneingangsphase in Gruppen organisierten. Es sei aus ihrer Sicht schwierig, mit Studierenden aus bereits „homogenen Gruppierungen" (Z. 368) in Kontakt zu treten und somit in „stabilen“ Gruppenkonstellationen studieren zu können. So führt A. Sanda hierzu an: „Die Gruppen haben sich schon spontan gebildet" (Z. 506). Da sie das Studium über die Regelstudienzeit hinaus verlängert habe, sei es nicht einfach, einer stabilen Gruppe anzugehören. Denn die Studienverlängerung bewirke eine Veränderung der ursprünglichen Studienkonstellation, was intensivere Beziehungen mit Blick auf die Zusammenarbeit mit Mitstudierenden erschwere, da sich diese bereits während der Studieneingangsphase gruppierten.

5.4 Mehrfachbelastung aufgrund Studien-, Arbeits- und Familienalltag: eine intersektionale Perspektive

Die Daten zu migrantischen Studentinnen aus der Pädagogik und Sozialer Arbeit verweisen auf interdependente Differenzlinien bspw. aufgrund von Migration und Geschlecht im Hinblick auf die Vereinbarkeit von Studium, Familien- und bezahlter Teilzeittätigkeiten. So berichtet die Pädagogikstudentin C. Durion, dass sie aufgrund der Vollzeitbeschäftigung sowie unflexibler Arbeitszeiten ihres Ehepartners für Care-Aufgaben 
zu Hause verantwortlich sei. Dies hat zur Folge, dass sie ihre längerfristige Studienplanung den familiären Verpflichtungen anpassen muss. Als besonders schwierig gestaltet sich die Vereinbarkeit des Studienalltags mit der Betreuung ihrer jüngeren Kinder. So sei sie aufgrund abendlicher Lehrveranstaltungen teilweise auf Betreuungsstrukturen angewiesen, die über die öffentlichen Kinderbetreuungszeiten hinausgehen. Wenn sie also abends um halb sieben noch Lehrveranstaltungen besuche, "hört die Betreuung für unsere Kleinste auf" (Z. 300). So schildert sie, dass ihre älteren Kinder die jüngeren Geschwister hin und wieder im Nachbarort abholen, was ihr Angst bereitet: „Jetzt ist es halt dunkel um diese Uhrzeit, das heisst die gehen da im Dunkeln ins Nachbardorf und holen da ihre Schwester ab und bringen sie nach Hause" (Z. 301-303). Des Weiteren thematisiert die Studentin finanzielle Engpässe, welche mit der externen Betreuung der Kinder entstehen. Aufgrund der Vollzeit-Berufstätigkeit ihres Partners und ihrem Teilzeitstudium können sie diese jedoch zeitlich bedingt nicht kompensieren. Daher müssen sie „auf eine Betreuung zurückgreifen, aber [Gelder] möglichst klein halten" (Z. 89-90). Entsprechend ergeben sich für die Studentin neben finanziellen Engpässen aufgrund von externen Betreuungskosten für die Kinder durch das Teilzeitstudium und Care-Aufgaben zeitliche Restriktionen. Diese Konstellation verursacht, dass sie das Familieneinkommen durch die Lehrtätigkeit an einem Gymnasium zwar zu verbessern beabsichtigt, dies jedoch wegen eines ausgeschöpften Studien- und Familienalltags nur begrenzt möglich ist. Insofern hat sich weder ihre finanzielle Situation dadurch verbessert, noch hat sie im Studien- und Familienalltag dadurch eine Entlastung erfahren. Dieses Dilemma, Teilzeitarbeit mit Studium und Familienarbeit zu vereinbaren, artikuliert C. Durion wie folgt: „Ich komme zwei volle Tage her [Hochschule] (...). Ich bin dienstags noch zwei Stunden in der Schule und unterrichte, und bin ansonsten zuhause. Ich habe natürlich keine freie Minute (...), es muss gekocht werden, es muss eingekauft werden, es muss gewaschen werden, es muss geputzt werden. Ich hab eigentlich immer gearbeitet, ich hatte nie wirklich viel Zeit für mich" (Z. 186-197).

Die Feminisierung von Care-Arbeit findet darin Ausdruck, dass diese mehrheitlich von Frauen erbracht wird (EBG 2010: 11), Frauen demnach aber auch stärker von prekären Verhältnissen betroffen sind. Aus intersektionaler Perspektive verdeutlicht das Beispiel von C. Durion mit Blick auf ihren Studien-, Arbeitsund Familienalltag, dass die Dreifachbelastung durch die unentgeltliche (Re-)Produktionsarbeit, also Care-Arbeit, zur (weiblichen) Prekarisierung beiträgt (Gutiérrez Rodríguez 2014) und im Fall der Studentin weder durch finanzielle noch zeitliche Ressourcen aufgrund des Studiums kompensiert werden können. C. Durion versucht, die Mehrfachbelastung im Studien-, Arbeits- und Familienalltag durch eine Verlängerung der Regelstudienzeit teilweise $\mathrm{zu}$ entschärfen, um somit ihre Studienplanung „familienfreundlicher“ $\mathrm{zu}$ gestalten. Auch wenn sich der zeitliche Mehraufwand durch die Studienverlängerung nicht ausgleichen lässt, ist sie zuversichtlich, dass die Hochschule ihren Antrag genehmigen wird, wie dies bei einer Kommilitonin bereits der Fall gewesen sei.

Obwohl die Belastung durch das Studium zugenommen habe, nimmt die Studentin die Atmosphäre an der Pädagogischen Hochschule positiv wahr. Um die verschiedenen Logiken von hochschulischem Studienalltag, Familie und Teilzeittätigkeit zu einem funktionierenden Ganzen zusammenzufügen, gehe sie sehr strukturiert vor und benutze jede freie Minute in der Pause oder in der Bibliothek für Studienzwecke. Freie Zeit sei für sie kostbar, weshalb sie wenig Kontakt zu Mitstudierenden pflege. Die Lebenssituation von Studentinnen mit familiären und beruflichen Verpflichtungen wirkt sich unmittelbar auf das Studium aus, sei es durch finanzielle und zeitliche Einschränkungen oder durch eingegrenzte Flexibilität, so bspw. hinsichtlich Studienplanung und -organisation, Studienort, Praktika etc. Wie die Erzählung von C. Durion illustriert, stehen Studierende mit Kindern vor besonderen Anforderungen, ihr Studium zu meistern und ihrer Familie gerecht werden zu können. In einer Ausnahmestellung gegenüber ihren kinderlosen Mitstudierenden haben diese Studentinnen keine gleichberechtigte Teilhabe an der hochschulischen Ausbildung (Middendorff 2007).

5.5. Agency im Kontext eines Fachhochschulstudiums: Die Perspektive migrantischer Studentinnen und Studenten

Migrantische Studierende haben unterschiedliche Handlungsstrategien entwickelt, um mit bestehenden Herausforderungen und Benachteiligungen an der Hochschule umzugehen. Die bisher erhobenen Daten verweisen darauf, dass die Studierenden trotz unterschiedlicher Ressourcen und Lebenssituationen zielorientiert gegenwärtige Herausforderungen aushandeln. Die hier angeführten Beispiele lehnen sich an 
qualitativ-empirische Studien zum Thema Bildungserfolge migrantischer Studierenden an, die den Blick auf die Perspektivenvielfalt der Studierenden und ihrer individuellen Handlungsfähigkeit bei der Bewältigung unterschiedlicher Hürden im Studium schärfen (Schwendowius 2015; Tepecik 2011).

Handlungsfähigkeit resp. agency bedeutet die Fähigkeit, spezifische Problemlagen aus eigenen Ressourcen zu lösen. Tran (2017) unterscheidet in ihrer Studie fokussierend auf internationale Studierende an australischen Universitäten zwischen „individual agency“ resp. Selbstbefähigung und „collective agency“, was die Fähigkeit beinhaltet, ein Netzwerk aufzubauen, um sich gegenseitig zu unterstützen (ebd.: 399-400). Auf beide Ansätze wird in folgenden Ausführungen Bezug genommen.

In den Interviews mit Studierenden der Sozialen Arbeit, aber auch der Pädagogik erwecken diese den Anschein, im Studium und als berufstätige Sozialarbeiter*innen und Pädagog*innen auf ein bestimmtes Ziel hinzuarbeiten. Dies kommt insbesondere darin zum Ausdruck, dass sie stets abzuwägen haben, was mit Blick auf den beruflichen Werdegang nützlich ist. Dies zeigt sich bspw. in der Pflege und Kultivierung eines persönlichen Netzwerks an der Hochschule. Die Studentin M. Aredhi unterhält ausschließlich Kontakte mit Studierenden, welche wie sie praxisbegleitend studieren und sich in einer ähnlichen Situation befinden. Sie legt Wert darauf, das bereits vorhandene Netzwerk mit Weggefährt*innen zu pflegen. $\mathrm{Zu}$ entscheiden, was Sinn macht, ist in ihrem Fall auch vor dem Hintergrund vorhandener Zeitressourcen zu betrachten, was hinsichtlich ausgefüllter Studien- und Arbeitswochen nachzuvollziehen ist. So macht sie die Erfahrung, dass im Rahmen von kollektiven Lernarrangements nicht, wie beabsichtigt, Aufgaben bearbeitet werden, sondern einzelne Personen in der Gruppe Alkohol trinken. Deshalb habe sie den Entschluss gefasst, sich nicht mehr mit Lerngruppen zu verabreden, da sie einfach „nicht immer in allem den $\operatorname{Sinn}^{\text {" }}$ (Z. 380) erkenne.

Was die Handlungsfähigkeit betrifft, schreibt Giddens (1997: 79), Menschen seien sich immer darüber im Klaren, was sie tun. Allerdings würden sie nicht wissen, was ihrem Handeln folge. Die befragte Studentin hätte "schon sehr früh gewusst" (Z. 32) resp. in der Adoleszenz, wie sie sich beruflich betätigen würde. Diese Einsicht unterstreicht Giddens' Argument, Menschen handeln „intentional“, sprich nicht in der Absicht etwas zu tun, sondern unter der Aus- sicht, etwas zu tun (ebd.). Die Studentin bringt dies wie folgt zum Ausdruck: "Ich gehe nicht einfach schaffen, sondern ich habe auch einen Auftrag, ich habe eine Haltung, habe eine Rolle. Es ist nicht so, dass ich einfach einmal schaffen gehe und schaue, ob es mir passt. " ( $Z$. 257-260).

Die Fähigkeit also, personelle und sachliche Entscheidungen in Bezug auf das Privatleben, Studium und Beruf zu treffen, kann als Handlungsstrategie zur Bewältigung spezifischer Herausforderungen im Hochschulalltag betrachtet werden.

Die Ausführungen der Studentin S. Erdal verdeutlichen eine weitere Form der Kompetenz, sich auf der Basis eigener Ressourcen zu befähigen. Bspw. kämpft sie seit Beginn des Studiums um die Anerkennung von geleisteten Arbeitsstunden in verschiedenen Handlungsfeldern der Sozialen Arbeit. Sie fühlt sich benachteiligt, weil die zuständige Fachstelle des Bachelor-Studiums ihre von Mehrfachbelastung beeinflusste Lebenssituation als Studentin, berufstätige Sozialarbeiterin und Alleinverantwortliche eines Zwei-Personenhaushalts nicht berücksichtigt.

Obwohl die Hochschule S. Erdal mit dem Entscheid hinhält, entwickelt sie im Kontext hochschulischer Anforderungen eine transformative Kraft zwischen Anpassung und Verhandlung. Sie äußert sich dazu wie folgt: „Und ich hab einen Antrag gestellt und der wurde abgelehnt. Dann habe ich noch einmal versucht (...), also an die Leitung habe ich wieder einen Antrag gestellt und sie haben wieder abgelehnt mit der Begründung, dass ich die Voraussetzungen nicht erfülle, $d a$ ich anstelle von sechs, eine fünfeinhalbjährige Praxiserfahrung nachweisen konnte, was meiner Ansicht nach paradox ist" (Z. 218-222).

Anzeichen einer transformativen Kraft zeigen sich daran, dass S. Erdal auch nach einem Negativentscheid nicht aufgibt, sondern erneut das Gespräch mit unterschiedlichen Verantwortlichen der Hochschule sucht: „Ich hab nicht aufgehört. (...) und dann habe ich vor kurzem mit dem Verantwortlichen noch einmal ein Gespräch gehabt und (...) in einem Gesuch geschrieben, dass ich wegen meinen finanziellen Möglichkeiten kein Praktikum absolvieren kann" (Z. 225-235).

In Anbetracht der Entwicklung von agency im Rahmen institutioneller Anforderungen zeigt das Beispiel S. Erdals, dass sie durch mehrmaliges Anschreiben und persönliche Absprachen versucht, auf hochschulische Entscheidungsprozesse Einfluss zu nehmen. Dadurch entwickelt sie Verhandlungsfähigkeit, die sie gezielt einzusetzen versteht. Allerdings 
erfordert der Umgang mit institutionellen Rahmenbedingungen besondere Anstrengungen und Beharrlichkeit.

\section{Zielgerichtetes Handeln wider kategoriale Zuweisungen an der Hochschule: ein Fazit}

Geschlecht, Migration und nationale Herkunft stellen in den hier untersuchten Daten artikulierte Dimensionen dar. Dies wird einerseits in Zusammenhang mit Deutsch resp. Französisch als Zweitsprache von Studierenden mit „Migrationshintergrund“ thematisiert, den sprachlichen Anforderungen zu genügen. Seitens migrantischer Studentinnen und Studenten wird erwartet, dass sie die Schriftsprache beherrschen und gleichzeitig die Schweizer Dialekte verstehen, was sie als herausfordernd und teilweise ausgrenzend erleben. Zumal die Sprache im Kontext von Migration häufig als Unterscheidungs- resp. Distinktionsmerkmal fungiert, lassen die Erzählungen hierzu hierarchische Zugehörigkeiten entlang von Sprachkenntnissen erkennen. So verdeutlichen die Aussagen der hier befragten Studierenden mit „Migrationshintergrund“ explizite oder subtile Formen von Ausgrenzungen im Kontext des Fachhochschulstudiums, welche insbesondere in Gruppenprozessen zum Ausdruck kommen. Denn auch im Kontext der Sozialen Arbeit und der Pädagogik werden heterogene Lebenswelten eher als Konfliktquelle denn als Ressource gedeutet. Die Daten zeigen, dass die Erfahrungen und biografischen Ressourcen migrantischer Studierender nicht als solche ausgelegt werden und sie eher von Differenzmarkierungen entlang dualistischer Konstruktionslogiken, wie bspw. „Einheimische - MigrantInnen“, „Mehrheit - Minderheit“ bzw. als „Migrationsandere“ - charakterisiert werden.

Die Ergebnisse einer Untersuchung an der Fachhochschule Kiel unterstützt die Annahme, dass die Sprache im Kontext von Migration aufgrund von Normalitätsvorstellungen - auch im universitären Kontext - als Unterscheidungs- resp. Distinktionsmerkmal fungiert. So werden sprachliche Schwierigkeiten - insbesondere in monolingual ausgerichteten Bildungsinstitutionen - als Problemfeld resp. „als mangelnde fachliche Kompetenz oder ,Dummheit' bewertet " (Discher/Plösser 2010: 9). Die befragten Studierenden geben an, dass Sprache als Mittel zur Herstellung von Normalität und Differenz genutzt wird und ihre Partizipation in der Hochschule u. a. aufgrund der unzureichenden Anerkennung ihrer mehrsprachigen Kompetenzen prekär bleibt (ebd.).
Des Weiteren bewirkt die einseitige Fokussierung auf die Sprache, dass Studierende auf ihre nationale Herkunft reduziert werden. So verdeutlichen Aussagen von migrantischen Studierenden subtile Formen von Ausgrenzungen insbesondere in Gruppenbildungsprozessen und im Rahmen von Lehrveranstaltungen mittels direkter Adressierung, sei dies als „sprachgewandte“ „Migrationsandere“ oder umgekehrt als solche mit angeblichen Sprachdefiziten.

Migrantische Studierende müssen sich eher mit Zuschreibungen auseinandersetzen, die auf geschlechterdifferenten Stereotypen von Weiblichkeit und Männlichkeit in Wechselwirkung mit Vorstellungen von traditionellen und patriarchalen Weiblichkeitskonzepten bei Zugewanderten gründen. So betont Ehlert, dass das Konstrukt der „Eingewanderten“ die einer „anderen“ Kultur angehörig und deshalb fremd und hilfsbedürftig seien, bis heute die Vorstellung vieler Sozialarbeitenden prägen. Medien, Forschung, Politik und Alltagspraxis reproduzieren und verfestigen diese stereotypen Bilder (Ehlert 2012: 68 u. 74). Sie betrachtet deshalb die Auseinandersetzung mit Prozessen der Konstruktion von „Fremdem“ und Differenzen zwischen Generationen und Geschlechtern als grundlegend in der hochschulischen Ausbildung. „Dazu zählt auch die Auseinandersetzung mit Migration und Geschlecht, denn Geschlecht, als sozialer Platzanweiser und als Container für geschlechtsbezogene, kulturelle Zuschreibungen und Stereotypien läuft in allen Prozessen immer mit" (ebd.: 69f.). Ebenso wird deutlich, dass migrantische Studierende teilweise selbst an geschlechtlichen Zuschreibungspraktiken beteiligt sind.

Migrantische Studentinnen mit familiären Verpflichtungen berichten über eine erschwerte Vereinbarkeit von Familie, Studium und Teilzeittätigkeit und die Notwendigkeit eines großen Organisationsvermögens, um das Hochschulstudium zu bewältigen. Durch zielgerichtetes Handeln angesichts ausgelasteter Studien- und Lebenskonstellationen gilt es ihrerseits, die Teilnahme und folglich die Relevanz von Lerngruppenarrangements abzuwägen. Auch lässt sich zielgerichtetes Handeln einer Studentin daran erkennen, dass sie sich trotz Mehrfachbelastung dezidiert für die Anerkennung von Praxisleistungen einsetzt, obwohl institutionelle Regelkonformitäten seitens der Hochschule ihrem Anliegen entgegenwirken.

Die Aussagen von migrantischen Studierenden verweisen darauf, dass Gefühle des Integriert-Seins, welche in Gruppenprozessen entstehen, angesichts ausgefülltem Studien- und Praxisalltags in Frage gestellt werden. 
Herausforderungen, die mit Ungleichheitsdimensionen in Zusammenhang stehen, welche die Situation einzelner Studierender betreffen, wie bspw. Migration bzw. nationale Herkunft und Geschlecht sowie Sprache, werden im Studium der Sozialen Arbeit und der Pädagogik in der Regel nicht explizit aufgegriffen. Zwar verdeutlichen die im Rahmen der Interviews artikulierten Erfahrungen der Studierenden, dass die betreffenden Hochschulen mit Flexibilität, Unterstützungs- und Beratungsangeboten auf die Anliegen und Bedürfnisse der Studierenden eingehen, was ihrerseits hochgeschätzt wird. Dennoch verweisen ihre Erfahrungen auch darauf, dass bestimmte Konventionen, die lebensweltliche Vielfalt im Curriculum sowie im hochschulischen Kontext gezielt angehen und Studierende mit unterschiedlichen Bedingungen sowie Differenzerfahrungen entsprechend berücksichtigen würden, teilweise fehlen. Einzelne Befragte unterstreichen zwar, dass Grundsätze der Nicht-Diskriminierung für die Hochschulkultur ein äußerst wichtiges Anliegen darstellten. So legten die Studienleitungen als auch Dozierende großen Wert auf eine pluralistische Hochschulkultur und bemühten sich, solche Grundsätze zu verwirklichen. Allerdings lässt sich aus den Daten zugleich ableiten, dass eine diversitätssensible Programmatik, u. a. mit dem Ziel, Differenzmarkierungen und Ausschlussrisiken sowie Erfahrungen prekärer Zugehörigkeit anzugehen resp. zu verhindern, noch nicht vorliegt. Dies obwohl im Zuge der Transformationsprozesse der (Fach-)Hochschulbildung der Diskurs über Diversität seit einigen Jahren Einzug gehalten hat. So verzeichnen die Hochschulen - auch in der Schweiz - u. a. verschiedene Aktionspläne, welche die bereits bestehenden Ansätze zur Gleichstellung der Geschlechter durch „Diversity Policies“ ergänzen und der soziokulturellen Vielfalt von Mitarbeitenden und Studierenden Rechnung tragen sollen. Mit diesen neuen Gleichstellungsansätzen gelangen neben Geschlecht weitere Ungleichheits- und Differenzkategorien in den Fokus, wie bspw. Nationalität resp. Herkunfts- und Migrationshintergrund. Mit diesen „Diversity Policies“ und Leitsätzen bekennen sich (Fach-)Hochschulen zur Anerkennung von Vielfalt und Heterogenität als gesellschaftliche Ressource wie auch als Qualitätsmerkmal von Bildungseinrichtungen sowie zur Umsetzung der rechtlichen Antidiskriminierungsgebote. Gerade im Hinblick auf deren Umsetzung würden sich für Hochschulen verschiedene innovative Ansätze bieten, tradierte Wissens- und Machtordnungen zu durchdenken und differenz- bzw. zuschreibungsre- flexive Perspektiven in die Hochschulbildung einzubringen. Entsprechende Ansätze implizieren sowohl die Frage, wie Differenzverhältnisse im Hochschulkontext kritisch beleuchtet werden können, als auch die Bereitschaft, entsprechenden Ausgrenzungsprozessen auf der symbolischen und strukturellen Ebene entgegenzutreten. Die besondere Herausforderung dabei dürfte darin liegen, angesichts der Vielfalt unterschiedlicher Diversitätsdimensionen eine Strategie bzw. konkrete Formen der Umsetzung zu erarbeiten, die über Normalisierungsprozesse hinausgehen und normative sowie strukturell hervorgebrachte Differenzmarkierungen aufgreift, die die Lebensbedingungen von Menschen prägen. Denn die Fokussierung auf bestimmte Differenzdimensionen resp. Personengruppen und folglich die Subdisziplinbildung (Interkulturelle Pädagogik, Alterspädagogik, Integrationspädagogik etc.) wird aufgrund des Zuordnungscharakters dieser Personengruppen u. a. von Kritiker*innen der Interkulturellen Erziehungswissenschaft in Frage gestellt (Breinbauer 2008: 70). Demnach werden in der Literatur Lösungsansätze diskutiert, die „über den Begriff der Anerkennung des Anderen (...) führt, diesen aus seiner umgangssprachlichen Formlosigkeit herausführt und auf seine Leistungsfähigkeit als ethisch-moralische Kategorie, und das heißt auch, auf seine impliziten Voraussetzungen prüft. Die Besonderheit dieses Vorschlages liegt darin, dass die Auseinandersetzung mit der Andersheit und Fremdheit des Anderen nicht durch Assimilation verharmlost werden kann. In der Konsequenz könnte ,Diversity' das Identitätsdenken schwächen, indem Identitätspositionen relativiert und zugleich durch Identitäts-Vervielfältigung gestärkt werden (...)“ (ebd.). Ein solcher Ansatz dürfte es ermöglichen, Vielfalt und Differenz im Kontext eines (Fach-)Hochschulstudiums über Normalitätsvorstellungen und Identitätsfixierungen hinweg zu betrachten und folglich aus einer kritischen resp. offeneren Perspektive anzugehen.

Daraus ergibt sich die Notwendigkeit, Vielfalt und Differenz als Querschnittsthemen curricular zu verankern sowie auf unterschiedlichen Ebenen, Fachbereichen und Organisationseinheiten anzugehen. Dazu ist die Sensibilisierung der Studierenden und Hochschulmitarbeitenden - Mitglieder des Hochschulmanagements, Führungskräfte und Mitarbeitende in den Bereichen Aus- und Weiterbildung etc. - erforderlich. 


\section{Literatur}

Attia, I. (2014): Rassismus (nicht) beim Namen nennen. Aus Politik und Zeitgeschichte, 64 (13-14), 8-14.

Banscherus, U./Himpele, K./Staack, S. (2011): Die soziale Dimension: Der blinde Fleck im Bologna-Prozess. Online: http://www.hof.uni-halle.de/journal/texte/11_1/Banscherus.pdf [26.11.2018].

Becker, R. (Hg.) (2011): Integration durch Bildung. Bildungserwerb von Migranten in Deutschland. Wiesbaden: VS Verlag.

Bieri Buschor, C./Berweger, S./Keck Frei, A./Kappler, C. (2014): Projektbericht GUNST - Geschlechts(un)typische Studienwahl: Weshalb Frauen Ingenieurwissenschaften studieren und Männer Primarlehrer werden, Pädagogische Hochschule Zürich, Abteilung Forschung und Entwicklung, Forschungsgruppe Professionalisierung und Kompetenzentwicklung. Online: https://www.phzh.ch/ MAPortrait_Data/161973/9/Projektbericht_GUNST.pdf [21.07.2017].

Blumer, F. (2016): Die Mär von der Chancengleichheit. Unipress, $168,26-27$.

Bourdieu, P. (1990): Was heißt sprechen? Zur Ökonomie des sprachlichen Tausches. Wien: Braumüller.

Bourdieu, P./Passeron, J. C. (1971): Die Illusion der Chancengleichheit. Untersuchungen zur Soziologie des Bildungswesens am Beispiel Frankreichs. Stuttgart: Ernst Klett.

Breinbauer, I. M. (2008): Pädagogik auf unsicherem Terrain: Die Vielfalt der Differenzen und die Unsicherheit über Weg und Ziel ihrer Bearbeitung als Herausforderung für Diversity-Pädagogik. In: Iber, K./Virtbauer, B. (Hg.): Diversity Management. Eine transdisziplinäre Herausforderung. Göttingen: Vienna University Press, 69-81.

Buchmayr, M./Neissl, J. (Hg.) (2006): Work-Life-Balance und Wissenschaft - ein Widerspruch? Münster: LIT.

Bundesamt für Statistik BFS (2015): Studien- und Lebensbedingungen an den Schweizer Hochschulen. Online: https://www.bfs.admin.ch/bfs/de/home/statistiken/ bildung-wissenschaft/personen-ausbildung/sozialewirtschaftliche-lage-studierenden.assetdetail.34946o. html [05.12.18].

Bundesamt für Statistik BFS (2017): Studien- und Lebensbedingungen an den Schweizer Hochschulen. Online: https://www.bfs.admin.ch/bfs/de/home/statistiken/kataloge-datenbanken/publikationen.assetdetail.3822341.html [29.11.2018].

Burger, H./Pfaff-Czarnecka, J./Pielage, P. (2013): Heterogenität an der Universität - Studieren mit Migrationshintergrund. Skizze eines Forschungsprojekts. In: Brandl, H./Arslan, E./Langelahn, E./Riemer, C. (Hg.): Mehrsprachig in Wissenschaft und Gesellschaft. Mehrsprachigkeit, Bildungsbeteiligung und Potenziale von Studierenden mit Migrationshintergrund. Online: http://biecoll. ub.uni-bielefeld.de/volltexte/2013/5274/index_de.html [22.09.2017].

Crenshaw Williams, K. (1994): Mapping the Margins: Intersectionality, Identity Politics, and Violence Against Wo- men of Color. In: Fineman, M. A./Mykitiuk, R. (Hg.): The Public Nature of Private Violence. New York: Routledge, 93-118.

Discher, K./Plößer, M. (2010): Erfahrungen von Studierenden mit Migrationshintergrund an der Fachhochschule Kiel. Ergebnisbericht. Online: http://www.fh-kiel. de/fileadmin/data/technologietransfer/institut_frauenforschung/pdfs/Ergebnisbericht_fertig1._doc.pdf [22.09.2017].

Dorlin, E. (Hg.) (2010): Sexe, race, classe, pour une épistémologie de la domination. Paris: PUF.

Ehlert, G. (2012): Gender in der Sozialen Arbeit. Konzepte, Perspektiven, Basiswissen. Schwalbach/Ts.: Wochenschau-Verlag.

Eidgenössisches Büro für die Gleichstellung von Mann und Frau EBG (2010): Anerkennung und Aufwertung der Care-Arbeit. Impulse aus Sicht der Gleichstellung. Online: https://www.ebg.admin.ch/dam/ebg/de/dokumente/ care/anerkennung_und_aufwertungdercare-arbeit.pdf. download.pdf/anerkennung_und_aufwertungdercarearbeit.pdf [23.11.18].

European Commission (2012): She Figures 2012. Gender in Research and Innovation. Statistics and Indicators. Directorate-General for Research and Innovation. Online: http:// ec.europa.eu/research/science-society/document_library/pdf_06/she-figures-2012_en.pdf [29.11.18].

Falquet, J. (2010): La règle du jeu. Repenser la co-formation des rapports sociaux de sexe, de classe et de „race“ dans la mondialisation néoliberale. In: Dorlin, E. (Hg.): Sexe, race, classe, pour une épistémologie de la domination. $\mathrm{Pa}-$ ris: PUF, 71-90.

Fellenberg, F. (2011): Gilt Frauenförderung bei Studierenden als unfair? Eine Befragung zur Akzeptanz von Gleichstellungsmaßnahmen bei Studierenden in Deutschland und der Schweiz. GENDER, 1, 117-125.

Felouzis, G./Charmillot, S. (2017): Schulische Ungleichheit in der Schweiz. Social Change in Switzerland. Online: http:// socialchangeswitzerland.ch/?p=1096 [26.11.18].

Foucault, M. (1977): Der Wille zum Wissen. Sexualität und Wahrheit. Erster Band. Frankfurt a. M.: Suhrkamp.

Giddens, A. (1997): Die Konstitution der Gesellschaft. Grundzüge einer Theorie der Strukturierung. Frankfurt/New York: Campus Verlag.

Gisbert, K. (2001): Geschlecht und Studienwahl. Biographische Analysen geschlechtstypischer und -untypischer Bildungswege. Münster: Waxmann.

Gutiérrez Rodríguez, E. (2011): Intersektionalität oder: Wie nicht über Rassismus sprechen? In: Hess, S./Langreiter, N./Timm, E. (Hg.): Intersektionalität revisited. Empirische, theoretische und methodische Erkundungen. Bielefeld: transcript, 77-100.

Gutiérrez Rodríguez, E. (2014): The Precarity of Feminisation. On Domestic Work, Heteronormativity and the Colonality of Power. International Journal of Politics, Culture and Society, 27 (2), 191-202. 
Hadjar, A./Hupka-Brunner, S. (Hg.) (2013): Geschlecht, Migrationshintergrund und Bildungserfolg. Weinheim/Basel: Beltz.

Hering, S./Kruse, E. (2004): Frauen im Aufwind des Bologna-Prozesses? Erste Hinweise zu Chancen, Risiken und Nebenwirkungen. Eine Tagungsdokumentation: „Analysen und Handlungsempfehlungen zur Herstellung von Chancengleichheit bei der Einführung von Bachelor- und Masterstudiengängen an deutschen Hochschulen" am 1./2. Oktober 2003 in Frankfurt am Main.

Kalpaka, A. (2002): Heterogenität und Homogenisierungsdruck. Anforderungen an das professionelle Handeln in der Einwanderungsgesellschaft. Supervision. Mensch Arbeit Organisation, 4, 38-43.

Kalpaka, A. (2009): Institutionelle Diskriminierung im Blick - Von der Notwendigkeit Ausblendungen und Verstrickungen in rassismuskritischer Bildungsarbeit $\mathrm{zu}$ thematisieren. In: Scharathow, W./Leiprecht, R. (Hg.): Rassismuskritik. Band 2: Rassismuskritische Bildungsarbeit. Schwalbach/Ts.: Wochenschau-Verlag, 25-40.

Klinger, C./Knapp, G.-A./Sauer, B. (Hg.) (2007): Achsen der Ungleichheit. Zum Verhältnis von Klasse, Geschlecht und Ethnizität. Frankfurt/New York: Campus Verlag.

Knappik, M./Dirim, 1. (2013): „Native-Speakerism“ in der Lehrerbildung. Journal für LehrerInnenbildung, 3, 20-23.

Knappik, M./Dirim, 1./Döll, M. (2013): Migrationsspezifisches Deutsch und die Wissenschaftssprache Deutsch: Aspekte eines Spannungsverhältnisses in der LehrerInnenausbildung. In: Vetter, E. (Hg.): Professionalisierung für sprachliche Vielfalt. Perspektiven für eine neue LehrerInnenausbildung. Band 9. Baltmannsweiler: Schneider Verlag, 42-61.

Krüger, H.-H./Rabe-Kleberg, U./Kramer, R.-T./Budde, J. (Hg.) (2011): Bildungsungleichheit revisited. Bildung und soziale Ungleichheit vom Kindergarten bis zur Hochschule. Wiesbaden: VS Verlag.

Le Breton, M./Lichtenauer, A./Kita, Z. (2016): Zum Verhältnis von Gender und Migration im Fachhochschulkontext. Die Perspektive von Bachelor-Studierenden der Sozialen Arbeit. In: Kriesi, I./Liebig, B./Horwarth, I./ Riegraf, B.(Hg.): Gender und Migration an Universitäten, Fachhochschulen und in der höheren Berufsbildung. Münster: Westfälisches Dampfboot, 148-167.

Lenz, I. (2009): Geschlecht, Klasse, Migration und soziale Ungleichheit. Zur Differenzierung der Strukturkategorien: Zwischen illegalen MigrantInnen und Geschlechtsnomaden im postmodernen Milieu. In: Lutz, H. (Hg.): Gender Mobil? Geschlecht und Migration in transnationalen Räumen. Münster: Westfälisches Dampfboot, 52-68.

Liebig, B./Criblez, L./Gottschall, K./Levy, R./Sauer, B./SousaPoza, A. (2014): NFP 60 Gleichstellung der Geschlechter. Ergebnisse und Impulse. Synthesebericht. Online: http:// www.nfp6o.ch/SiteCollectionDocuments/NFP_6o_ Synthese_de_Web.pdf [29.11.18].

Lindt, I./Löther, A. (Hg.) (2008): Wissenschaftlerinnen mit Migrationshintergrund. Online: https://www.ssoar.info/ ssoar/handle/document/23342 [26.11.2018].
Lutz, H. (2007): „Die 24-Stunden-Polin“ - Eine intersektionelle Analyse transnationaler Dienstleistungen. In: Klinger, C./Knapp, G.-A./Sauer, B. (Hg.): Achsen der Ungleichheit. Zum Verhältnis von Klasse, Geschlecht und Ethnizität. Frankfurt/New York: Campus, 210-234.

Lutz, H./Herrera Vivar, M. T./Supik, L. (Hg.) (2013): Fokus Intersektionalität. Bewegungen und Verortungen eines vielschichtigen Konzepts. Wiesbaden: Springer VS.

Maihofer, A./Bergman, M. M./Hupka, S./Wehner, N./ Schwiter, K./Huber, E./Kanji, S. (2013): Kontinuität und Wandel von Geschlechterungleichheiten in Ausbildungsund Berufsverläufen. Schlussbericht zu Handen des Schweiz. Nationalfonds. Online: https://genderstudies. philhist.unibas.ch/fileadmin/user_upload/genderstudies/Dokumente/Forschung/Beruf-Erw13-06-06_NZZStyle_Summary_eingereicht.pdf [22.09.2017].

Majcher, A./Zimmer, A. (2010): Hochschule und Wissenschaft: Karrierechancen und -hindernisse für Frauen. In: Becker, R./Kortendiek, B. (Hg.): Handbuch Frauenund Geschlechterforschung. Theorie, Methoden, Empirie. Wiesbaden: VS Verlag, 705-712.

Mecheril, P. (2010): Migrationspädagogik. Hinführung zu einer Perspektive. In: Mecheril, P./Castro Varela, M. d. M./ Dirim, I../Kalpaka, A./Melter, C. (Hg.): Bachelor / Master: Migrationspädagogik. Weinheim/Basel: Beltz, 7-22.

Mecheril, P./Melter, C. (2010): Differenz und Soziale Arbeit. Historische Schlaglichter und systematische Zusammenhänge. In: Kessl, F./Plösser, M. (Hg.): Differenzierung, Normalisierung, Andersheit. Soziale Arbeit als Arbeit mit den Anderen. Wiesbaden: VS Verlag, 117-131.

Metz-Göckel, S. (2012): Theoretische Skizzen zur Hochschule in der Wissensgesellschaft. In: Klein, U./Heitzmann, D. (Hg.): Hochschule und Diversity. Theoretische Zugänge und empirische Bestandsaufnahme. Weinheim/Basel: Beltz, 46-68.

Middendorff, E. (2007): Lebenssituation Studierender mit Kind - Ausgewählte Befunde der Sozialerhebung des DSW und einer Online-Befragung des HISBUS-Panels. In: Cornelissen, W./Fox, K. (Hg.): Studieren mit Kind. Die Vereinbarkeit von Studium und Elternschaft: Lebenssituationen, Maßnahmen und Handlungsperspektiven. Wiesbaden: VS Verlag, 33-49.

Murdoch, J./Guégnard, C./Koomen, M./Imdorf, C./HupkaBrunner, S. (2014): Pathways to higher education in France and Switzerland. Do vocational tracks facilitate access to higher education for immigrant students? In: Goastellec, G./Picard, F. (Hg.): Higher Education in Societies - A Multi Scale Perspective. Rotterdam: Sense Publishers, 149-169.

Panagiotopoulou, A./Rosen, L./Wagner, M. (2016): „Die Frage ist halt jetzt, darf Pippi Langstrumpfs Vater noch der Negerkönig sein“. Zur (Re-)Produktion von Rassismus im Rahmen universitärer Lehrveranstaltungen. In: Arslan, E./Bozay, K. (Hg.): Symbolische Ordnung und Bildungsungleichheit in der Migrationsgesellschaft. Wiesbaden: Springer VS, 239-256. 
Polat, S./Wienand, C. (2014): ,Ceci n'est pas une Migrantin - Repräsentation, Migration und (Auto-)Biografie؛. In: Aced, M./Düzyol, T./Rüzgar, A./Schaft, C. (Hg.): Migration, Asyl und (Post-)Migrantische Lebenswelten in Deutschland: Bestandsaufnahme und Perspektiven migrationspolitischer Praktiken. Berlin: LIT, 157-176.

Pöllmann-Heller, K./Bitzan, R. (2017): Studie „MINTersectionality - Intersektionale Impulse für die Gender-Gleichstellungsarbeit an technisch orientierten Hochschulen". Teilstudie „Fokus Intersektionalität“. Online: https:// www.th-nuernberg.de/fileadmin/kompetenzzentren/ komgedi/Dokumente/MINTersectionality_INTER_Bericht_112017.pdf [19.02.2019].

Quehl, T./Mecheril, P. (2008): Unsere Sprache(n) sprechen. Offizielle Sprach- und Zugehörigkeitspolitiken in der Migrationsgesellschaft. Online: https://heimatkunde.boell. de/2008/03/01/unsere-sprachen-sprechen-offiziellesprach-und-zugehoerigkeitspolitiken-der [15.07.2017].

Riegel, C. (2016): Bildung - Intersektionalität - Othering. Pädagogisches Handeln in widersprüchlichen Verhältnissen. Bielefeld: transcript.

Saner, P./Vögele, S./Vessely, P. (2016): Schlussbericht. Art. School.Differences. Researching Inequalities and Normativites in the Field of Higher Art Education. Online: https:// blog.zhdk.ch/artschooldifferences/files/2016/10/ASD_ Schlussbericht_final_web_verlinkt.pdf [30.03.2018].

Scharenberg, K./Hupka-Brunner, S./Meyer, T./Bergman, M. M. (Hg.) (2016): Transitionen im Jugend- und jungen Erwachsenenalter. Ergebnisse der Schweizer Längsschnittstudie TREE. Zürich: Seismo.

Schwendowius, D. (2015): Bildung und Zugehörigkeit in der Migrationsgesellschaft. Biographien von Studierenden des Lehramts und der Pädagogik. Bielefeld: transcript.

Schwendowius, D./Thoma, N. (2016): Studienbiographien ,mit Migrationshintergrund'? Kritische Anmerkungen zu Praktiken der Besonderung in der universitären Praxis. $Z Q F, 17$ (1-2), 221-235.

Stamm, M. (2009): Migranten als Aufsteiger. Reflexion zum Berufserfolg von Auszubildenden mit Migrationshintergrund. Zeitschrift für Sozial- und Wirtschaftspädagogik, 3, 361-377.

Strauss, A./Corbin, J. (1996): Grounded Theory: Grundlagen Qualitativer Sozialforschung. Weinheim: Beltz.

Teubner, U. (2010): Beruf: Vom Frauenberuf zur Geschlechterkonstruktion im Berufssystem. In: Becker, R./Kortendiek, B. (Hg.). Handbuch Frauen- und Geschlechterforschung. Theorie, Methoden, Empirie. Wiesbaden: VS Verlag, 499-506.

Tepecik, E. (2011): Bildungserfolge mit Migrationshintergrund. Biographien bildungserfolgreicher MigrantInnen türkischer Herkunft. Wiesbaden: VS Verlag.

Tran, T. L. (2017): ,I am really expecting people to judge me by my skills': ethnicity and identity of international students. Journal of Vocational Education \& Training, 69 (3), 390-404.
Wetterer, A. (Hg.) (1995): Die soziale Konstruktion von Geschlecht in Professionalisierungsprozessen. Frankfurt a. M.: Campus Verlag.

Wetterer, A. (1999): Ausschließende Einschließung - marginalisierende Integration. Geschlechtskonstruktionen in Professionalisierungsprozessen. In: Neusel, A./Wetterer, A. (Hg): Vielfältige Verschiedenheiten. Geschlechterverhältnisse in Studium, Hochschule und Beruf. Frankfurt a. M.: Campus Verlag, 223-253.

Wimbauer, C. (1999): Organisation, Geschlecht, Karriere. Fallstudien aus einem Forschungsinstitut. Opladen: LeskeBudrich.

Winker, G./Degele, N. (2009): Intersektionalität. Zur Analyse sozialer Ungleichheiten. Bielefeld: transcript.

Witzel, A. (2000): Das problemzentrierte Interview [25 Absät$z e]$. Online: http://www.qualitative-research.net/index. $\mathrm{php} /$ fqs/article/view/1132/2519 [14.08.2017]. 